УДК 004.725.5

\title{
C.B. Сахарова
}

Одесская национальная академия пищевых технологий, учебно-научный институт холода, криотехнологий и экоэнергетики им. В.С. Мартыновского, ул. Дворянская, 1/3, Одесса, 65082

\section{НЕКОТОРЫЕ ОСОБЕННОСТИ ПРИМЕНЕНИЯ ЭКСПЕРТНЫХ СИСТЕМ ПРИ ПРОЕКТИРОВАНИИ ТЕЛЕКОММУНИКАЦИОННЫХ СЕТЕЙ}

\begin{abstract}
Выполненные исследования относятся к области проектирования телекоммуникаиионных сетей, в частности перспективных сетей доступа. Приведены результаты разработки экспертной системы позволяющая принять решение о целесообразном применении одной из технологий доступа, в частности рассмотрена технология DSL.

Ключевые слова: Сеть доступа - Параметры сетей доступа - Экспертная система Проектирование телекоммуникаиионных сетей
\end{abstract}

\section{C.B. Сахарова}

Одеська національна академія харчових технологій, навчально-науковий інститут холоду, кріотехнологій та екоенергетики ім. В.С. Мартиновського, вул. Дворянська, 1/3, Одеса, 65082

\section{ДЕЯКІ ОСОБЛИВОСТІ ЗАСТОСУВАННЯ ЕКСПЕРТНИХ СИСТЕМ ПРИ ПРОЕКТУВАННІ ТЕЛЕКОМУНІКАЦІЙНИХ МЕРЕЖ}

\begin{abstract}
Виконані дослідження відносяться до галузі проектування телекомунікаиійних мереж, зокрема перспективних мереж доступу. Наведено результати розробки експертної системи дозволяє ухвалити рішення про доиільний застосуванні однієї з технологій доступу, зокрема розглянута технологія DSL.

Ключові слова: Мережа доступу - Параметри мереж доступу - Експертна система Проектування телекомунікаиійних мереж
\end{abstract}

DOI: $10.15673 / 0453-8307.6 / 2014.31211$

This work is licensed under the Creative Commons Attribution International License (CC BY). http://creativecommons.org/licenses/by/4.0/

\section{І.ВВЕДЕНИЕ}

Характерной особенностью прогресса среди компьютерных информационных систем, актуальных в Украине, является безусловный приоритет разработок экспертных систем (ЭС), основанных на использовании искусственного интеллекта. Эта тенденция обоснована тем фактором, что экспертные системы дают возможность менеджеру или специалисту получать консультации экспертов по любым проблемам, о которых этими системами накоплены знания.

Под искусственным интеллектом обычно понимают способности компьютерных систем к таким действиям, которые назывались бы интеллектуальными, если бы исходили от человека.

Безусловно, решение специальных задач требует специальных знаний. Однако не каждая компания может себе позволить держать в своем штате экспертов по всем, связанным с ее работой, проблемам или даже приглашать их каждый раз, когда проблема возникла. Эта, на первый взгляд, сугубо экономическая проблема, что присуща, практически каждой молодой и небогатой стране, такой как Украина, явилась основной причиной следующего: главная идея использования технологии экспертных систем заключается в том, что- бы получить от эксперта его знания и, загрузив их в память компьютера, использовать всякий раз, когда в этом возникнет необходимость.

Являясь одним из основных приложений искусственного интеллекта, ЭС представляют собой компьютерные программы, трансформирующие опыт экспертов в какой-либо области знаний в форму эвристических правил (эвристик). Эвристики не гарантируют получения оптимального результата с такой же уверенностью, как обычные алгоритмы, используемые для решения задач в рамках технологии поддержки принятия решений. Однако часто они дают в достаточной степени приемлемые решения для их практического использования. Все это делает возможным использовать технологию экспертных систем в качестве советующих систем.

Сходство информационных технологий, используемых в экспертных системах и системах поддержки принятия решений, состоит в том, что обе они обеспечивают высокий уровень поддержки принятия бизнес-решений в Украине. Однако имеются три существенных различия, а вернее, особенности.

Первое связано с тем, что решение проблемы в рамках систем поддержки принятия решений отражает уровень ее понимания украинским кон- 
кретным пользователем, его ментальностью и способностью получить и осмыслить оптимальное решение. Технология ЭС, наоборот, предлагает пользователю принять решение, превосходящее его возможности.

Второе отличие указанных технологий выражается в способности ЭС пояснять свои рассуждения в процессе получения решения. Очень часто эти пояснения оказываются более важными для пользователя, чем само решение.

Третье отличие связано с использованием нового компонента информационной технологии общей эрудиции и знаний.

\section{II. ПОСТАНОВКА ЗАДАЧИ}

В рамках представленной работы решается задача разработки ЭС, позволяющей повысить эффективность проектирования и эксплуатации телекоммуникационных сетей и их сегмента - сетей доступа (СД). Одним из важнейших и сложных этапов является сбор и представление знаний в области проектирования телекоммуникационных сетей для обучения экспертной системы. Постановка задачи ограничена разработкой алгоритма создания ЭС и анализом теоретических аспектов проектирования интеллектуальной ЭС, основанной на знаниях. В качестве примера разработана ЭС, позволяющая принять решение о целесообразном применении одной из технологий доступа, в частности на первом этапе рассмотрены технологии $D S L$.

Целью работы является повышение эффективности проектирования и эксплуатации СД за счет разработки ЭС.

Объект исследования - сеть доступа.

Предмет исследования - методы разработки экспертных систем, методы представления знаний, методы проектирования сетей доступа.

\section{III. ЦЕЛЕСООБРАЗНОСТЬ РАЗРАБОТКИ ЭС ДЛЯ ПРОЕКТИРОВАНИЯ ТЕЛЕКОММУНИ- КАЦИОННЫХ СЕТЕЙ}

Украинский рынок услуг передачи данных в режиме on-line находится в начальной стадии своего развития. Основным сдерживающим фактором является несоответствие между большой себестоимостью услуг и платежеспособностью потребителей, в результате чего такого рода услуги до настоящего времени могли позволить себе только средние и крупные корпоративные пользователи. Не секрет, что для снижения себестоимости услуг важнейшую роль играет выбор среды передачи данных именно для организации "последней мили", то есть линий, по которым пользователи подключаются к точке доступу оператора. При построении сети, рассчитанной на массового пользователя, выбор технологии для "последней мили" становится принципиальным с точки зрения влияния на тарифы.

Современные концентраторы DSL представляют собой оборудование нового поколения, позволяет подключать абонентов к сети передачи данных, используя последние технологии, и имеет сетевые интерфейсы, такие как Ethernet, ATM,
$S D H$. Концентраторы устанавливаются в местах концентрации пользователей на стороне оператора связи и позволяют пользователям получать высокоскоростной доступ к сетям передачи данных, сохраняя при этом существующую инфраструктуру и доступ к ТфОП.

На сегодняшний день число производителей и поставщиков устройств подобного рода составляет несколько сотен, и выбрать нужное оборудование не так просто. Требования, которые предъявляет потребитель к разным классам $D S L$ оборудования, существенно различаются. Имеют значение: надежность, размеры, плотность портов, потребляемая мощность. Например, надежность концентраторов операторского класса должна быть значительно выше, чем концентраторов для кампусных приложений, где непродолжительные сбои в передаче трафика не столь критичны; то же касается и необходимого набора функций. Использование медной проводки и простая процедура установки концентратора делают начальные вложения для создания СД минимальными. Таким образом, использование концентраторов позволяет пользователям получать дополнительные виды услуг, а операторам - дополнительные виды дохода.

Вышесказанное подтверждает целесообразность разработки, экспертной системы, которая помогла бы операторам в выборе той или иной технологию $x D S L$, оборудования, вида кабеля и т.д., для построения "последней мили".

\section{IV. ПРИМЕР РАЗРАБОТАННОЙ ЭКСПЕРТ- НОЙ СИСТЕМЫ}

При разработке ЭС применено ПО Expek Pro версия 1.5+ для Windows, что является универсальной экспертной системой для оценки сложных объектов. Система обеспечивает качественное обоснование в задачах оценивания и принятия решения. Для формализации и решения конкретной оценочной задачи необходимо построить оценочную модель. Использована в Expek Pro оригинальная векторная модель представления знаний и механизм принятия решений вобрали в себя преимущества таких известных подходов, как нечеткая логика, нейронные сети, Байесовская оценка и другие.

Последняя миля - это довольно специфичный сегмент сети. Он должен иметь достаточно широкую полосу пропускания и при этом обеспечивать большую дальность без регенераторов. Данная экспертная система будет определять лучший вариант технологии $x D S L$ по заданным свойствам.

Основные компоненты информационной технологии экспертных систем: интерфейс пользователя, база знаний, интерпретатор, модуль создания системы.

Для ввода информации и команд в экспертную систему и получения выходной информации из нее менеджер (специалист) использует интерфейс. Команды включают в себя параметры, направляющие процесс обработки знаний. Информация обычно выдается в форме значений, присваиваемых определенным переменным. 
Менеджер может использовать четыре метода ввода информации: меню, команды, естественный язык и собственный интерфейс. Технология экспертных систем предусматривает возможность получать в качестве выходной информации не только решение, но и необходи-мые объяснения.

Пример сформированной базы данных объектов приведен на рисунке 1. Подробную информацию для анализа вклада свойств текущего объекта в его интегральную характеристику можно получить выполнив одиночный расчет. Пример отображения расчетов приведен на рисунке 2 .

Пример визуализации результатов анализа на основании базы знаний, которой обучена данная система, изображен на рисунке 3 .

Из данного графика видно, что наиболее приемлемой для анализируемого варианта исходных данных технология, является VDSL2.



Рисунок 1 - Пример сформированной базы данных

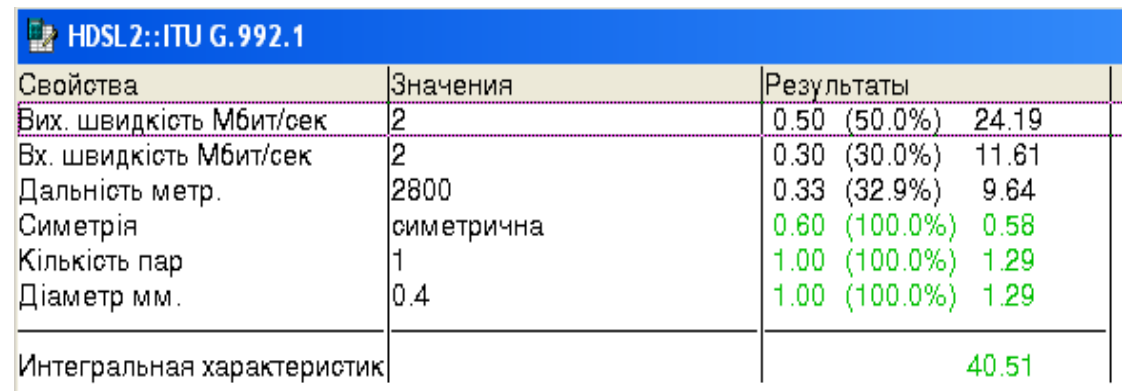

Рисунок 2 - Пример одиночного расчета

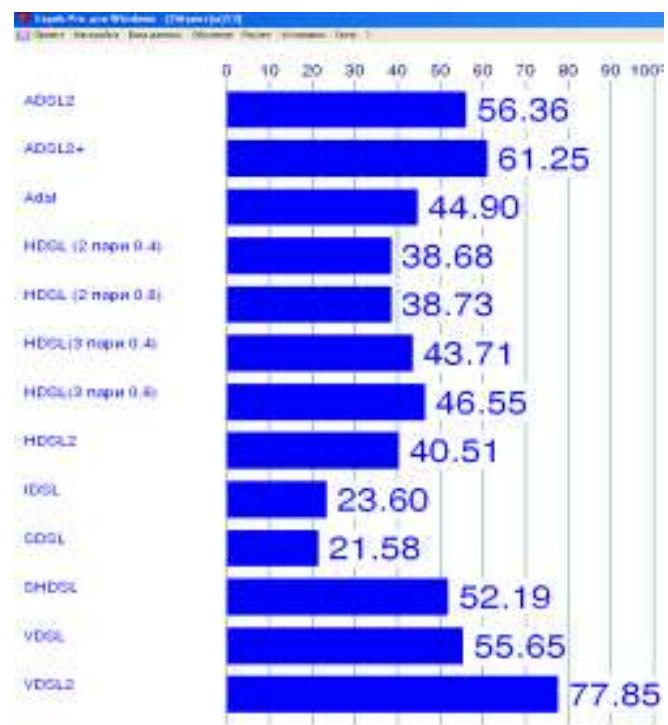

Puc. 3 - Пример визуализации результатов

\section{V. ВЫВОД}

В результате выполнения работы, результаты которой приведены в статье, был создан алгоритм разработки экспертной системы, которая предна- значена для первичного определения уровня качества сетей доступа с их свойствами и преимуществами, проведен анализ теоретических аспектов проектирования интеллектуальной экспертной системы, основанной на знаниях.

Проделанная работа формулирует задачи квалиметрии и помогает понять, как построить экспертную систему для рационального построения сетей доступа, а также включает пример разработки экспертной системы.

\section{ЛИТЕРАТУРА}

1. Сахарова С.В. Возможность применения экспертных систем при проектировании сетей доступа / С.В. Сахарова // Материалы Х МНТК «Математическое моделирование и информационные технологии»/ММИТ-2012/ - Одесса: ОГАХ. 2012. - C. 88.

2. Гайворонская Г. С. Оценка влияния некоторых факторов на процесс развития телекоммуникационных сетей / Г.С Гайворонская // Холодильна техніка і технологія. - 2006. -№2 (100). - С. 95-100.

3. Сахарова С.В. Задача выбора параметров сети доступа / С.В. Сахарова // Сборник тезисов III МТК «Проблеми телекомунікацій», КПІ, Киев, 21-24 апреля 2009. - С.61.; 
4. Гайворонская Г.С. Классификация параметров сетей доступа / Г.С. Гайворонская, С.В. Сахарова // Сборник тезисов Пятой МНТК «Современные информационно-коммуникационные технологии», Крым, Ялта, Ливадия, 05-09 октября 2009. - С.77-78.;

5. Сахарова С.В. Исследование параметров сетей абонентского доступа / С.В. Сахарова // Материалы VIII МНTК «Математическое моделирование и информационные технологии» /ММИТ-2008/ - Одесса: ОГАХ. -2008.-С. 29.

6. Гайворонська Г.С. Розробка експертної сис теми прогнозування вимог користувачів до телекомунікаційної мережі / Г.С. Гайворонська, О.М. Домаскін, С. В. Сахарова // I-th International Scientific Conference Information Technologies \& Interactions ITA 2014 Joint International Scientific Events on Informatics September 08-13, 2014, Kyiv, Ukraine.

7. Olena Domaskina, Oleg Domaskin Some features of information technology development of expert systems used in Ukraine // Natural Information Technologies. - Madrid: ITHEA, 2012. - P. 48-51.

\section{S.V. Sakharova}

Odessa National Academy of Food Technologies, Educational and Research Institute of Refrigeration, Cryotechnologies and Ecoenergetics n.a. V.S. Martynovskiy, 1/3 Dvoryanskaya str., Odessa, 65082

\section{SOME FEATURES OF EXPERT SYSTEMS' USAGE AT THE DESIGN OF TELECOMMU- NICATIONS NETWORKS}

The subject of the research, some results of which are presented in this paper, is focused on the development of modern telecommunication networks in Ukraine and allows solving the problems at the networks design and organization of access to infocommunication services. The chosen theme is closely related with the main directions of the world telecommunications development, which are defined in the main existing regulations and laws. A characteristic feature of computer information systems' progress, which are actual in Ukraine, is an absolute priority of expert systems' development. Questions of the expert systems' usage at the design and development of telecommunications networks in the modern world are considered in the paper.

The problem of expert system's development, which allows enhancing of the design and operation of telecommunication and access networks, is being solved within the given work. Statement of the problem is limited by the development of the algorithm for the expert system's development and analysis of the theoretical aspects of the knowledge based intelligent system's design. Done work formulates the qualimetry problems and helps to understand how to build an expert system for the access networks' rational development.

Expert system, allowing to choose proper access technology, is developed as an example. Particularly DSL technologies are considered at the first stage. The last mile is quite specific network's segment. It should have enough bandwidth while providing great transmission range without regenerators. This expert system will determine the best option of DSL technology on the basis of the desired properties. The system is developed with the usage of Expek Pro software (version 1.5+) for Windows.

The article consists of five sections - introduction, problem statement, the main part - the necessity of an expert system's development for the telecommunication networks' design, an example of the developed expert system and conclusions, as well as includes bibliography, used at the work writing.

Keywords: Access network - Parameters of access networks - Expert system - Design of telecommunication networks.

\section{REFERENCES}

1. Saharova S.V. 2012. Vozmozhnost' primenenija jekspertnyh sistem pri proektirovanii setej dostupa / S.V. Saharova // Materialy X MNTK «Matematicheskoe modelirovanie i informacionnye tehnologii»/MM IT-2012.-Odessa:OGAH. - 2012. - S.88 (in Russian). 2. Gajvoronskaja G. S. 2006. Ocenka vlijanija nekotoryh faktorov na process razvitija telekommunikacionnyh setej/ G.S Gajvoronskaja // Kholodil'na tehnika i tehnologija. - 2006. -№2 (100). - S. 95-100 (in Russian). 3. Saharova S.V. 2009. Zadacha vybora parametrov seti dostupa / S.V. Saharova // Sbornik tezisov III MTK «Problemi telekomunikacij», KPI, Kiev, 21-24 aprelja 2009. - C.61 (in Russian).

4. Gajvoronskaja G.S. 2009. Klassifikacija parametrov setej dostupa. G.S. Gajvoronskaja, S.V. Saharova. Sbornik tezisov Pjatoj MNTK «Sovremennye informacionnokommunikacionnye tehnologii», Krym, Jalta, Livadija, 0509 oktjabrja 2009. - C.77-78 (in Russian).
5. Saharova S.V. 2008. Issledovanie parametrov setej abonentskogo dostupa / S.V. Saharova // Materi-aly VIII MNTK «Matematicheskoe modelirovanie i informacionnye tehnologii» /MMIT-2008/ - Odessa: OGAH. 2008.-C. 29 (in Russian).

6. Gajvorons'ka G.S. 2014. Rozrobka ekspertnoï sistemi prognozuvannja vimog koristuvachiv do teleko-munikacijnoï merezhi / G.S. Gajvorons'ka, O.M. Do-maskin, S. V. Saharova // I-th International Scientific Conference Information Technologies \& Interactions ITA 2014 Joint International Scientific Events on Informatics September 08-13, 2014, Kyiv, Ukraine (in Russian).

7. O. Domaskina, O. Domaskin, 2012. Some features of information technology development of expert systems used in Ukraine // Natural Information Technologies. - Madrid: ITHEA, 2012. - P. 48-51 (in English).

Отримана в редакції 14.10.2014, прийнята до друку 17.11.2014 